\title{
Optimal Multiresolution Blending of Confocal Microscope Images
}

\author{
Hao-Chiang Shao, Wen-Liang Hwang ${ }^{\dagger}$, and Yung-Chang Chen \\ ${ }^{\dagger}$ Institute of Information Science, Academia Sinica, Taiwan \\ Department of Electrical Engineering, National Tsing Hua Univesity, Taiwan
}

\begin{abstract}
Typical mosaicing schemes assume that to-be-combined images are equally informative; thus, the images are processed in a similar manner. However, the new imaging technique for confocal fluorescence images has revealed a problem when two asymmetrically informative biological images are stitched during microscope image mosaicing. The latter process is widely used in biology studies to generate a higher-resolution image by combining multiple images taken at different times and angles. To resolve the above problem, we propose a multiresolution optimization approach that evaluates the blending coefficients based on the relative importance of the overlapping regions of the to-be-combined image pair. The blending coefficients are the optimal solution obtained by a quadratic programming algorithm with constraints that are enforced by the biological requirements. We demonstrate the efficacy of the proposed approach on several confocal microscope fluorescence images and compare the results with those derived by other methods.
\end{abstract}




\section{Introduction}

Confocal microscopes are now widely used in biological imaging because of the recent development of fluorescent probes and high-resolution imaging techniques. Large numbers of two- or three- dimensional images have been produced to visualize subcellular components in vivo; however, the images require post-processing to ensure proper interpretation, which creates new challenges for current image processing techniques [1]. An outstanding review of the major challenges that arise when processing fluorescence microscope images can be found in [2]. Because a microscope's field of vision is limited, biologists usually form a higher-resolution image by acquiring multiple images of a sample, taken at different times and from different angles, and then combine them via a mosaicing scheme.

Image mosaicing, which combines two or more images, is used in many fields, such as photogrammetry, computer vision, image synthesis, and computer graphics $[3,4,5,6,7,8,9]$. The process usually involves two phases: image registration and image blending. The former tries to identify the best blending position where the overlapping areas of the to-be-combined images are aligned, while the latter adjusts the intensity to obtain a smooth and seamless result. Some approaches try to combine both phases in a single framework; however, the operations are usually performed independently in the sense that most approaches try to optimize one phase without considering the other phase, or by assuming that the other phase has been solved. In this paper, we assume that the to-be-combined microscope images or image stacks have been registered by using one of many good registration algorithms that have been developed $[10,11,12,13,14]$. It is usually necessary to combine several images in microscope image mosaicing. Although a graph 
cut approach has been proposed to stitch multiple overlapping areas of several to-be-combined images [15], for simplicity, we propose a sub-optimal approach that combines two images at a time.

Most current blending algorithms assume that to-be-combined images are equally informative; thus, the images are processed in a similar manner [16]. However, this assumption may not hold for confocal microscope fluorescence images because the fluorescence intensity often attenuates rapidly and may deteriorate. The intensity degradation, which results from photobleaching of the overlapping region of the later-acquired image, means that the earlieracquired image is more informative for image mosaicing. Figure 1 shows the effect of photobleaching on multiple Drosophila brain images taken by confocal microscopes. The images in the leftmost column of the figure were acquired after the corresponding images in the same row in the middle column. Clearly, the overlapping regions, which are to the right of the dashed line of the images in the leftmost column, are darker than the corresponding regions to the left of the dashed line of the images in the middle column. Therefore, the issue of mosaicing this image pair is different to the traditional case because one of the two overlapping regions is less informative than the other.

Of the many image blending methods in use, Burt and Adelson's method [17] is the most popular because it is effective and can be implemented easily by using a fixed 3-tap blending vector and a pyramid structure [18], which is a multi-resolution representation of an image. A commonly used weighting sequence for blending two subband components is $0.25,0.5$, and 0.75 . More specifically, an image is decomposed into a series of subbands by REDUCE and EXPAND operations, as shown in Figure 3. The reduce operation ap- 
plies a low-pass filter and sub-samples by a factor of 2 in each dimension; and the expand operation up-samples by a factor of 2 in each dimension and applies a low-pass filter. In [19], an optimization approach is used to derive data-dependent weighting coefficients from the wavelet coefficients by solving two quadratic functions alternately. The approach requires that the derived weighting coefficients ensure that the area of the combined image affected by the blending process is smooth and retains as much information about the original images as possible. The blending approach proposed in this paper is a combination of the approaches in [17] and [19]. The subband coefficients in the Laplacian pyramid domain are blended by using the weighting coefficients derived by solving a constrained optimization problem. Our optimization algorithm is simpler than that in [19], and the information imbalance between the earlier- and later- acquired images is treated as a constraint. A simple optimization algorithm can be implemented easily and generalized by incorporating elements of the domain knowledge as constraints on the optimization problem.

We conducted experiments to compare the mosaicing results of the proposed method with those of Burt and Adelson's algorithm and the MosaicJ algorithm [16] for a set of images obtained by confocal microscope imaging. The results demonstrate that our mosaic images exhibit better local contrasts and smoothness.

\subsection{Intensity loss in microscope imaging}

The fluorescent intensity of microscope images suffers from several distortions. Because of the scattering and absorption of fluorescence, when an image stack is obtained by confocal microscopes, the intensity of the deeper 
layers in a specimen is usually lower, resulting in a loss of intensity along the $\mathrm{z}$ axis. Moreover, because of repeated imaging and the photobleaching effect, the contrast and brightness in the overlapping areas of a later-acquired image are lower. Most biological laboratories correct the contrasts of later-acquired images manually, but the approach is time-consuming and unreliable. Figure 2 shows that it is difficult to adjust the contrasts of later-acquired images to match those of the earlier-acquired images.

Other solutions have been proposed to compensate for intensity loss during microscopic imaging. The first solution utilizes biological methods. For example, it is now possible to make biological samples transparent to about 500 micrometers with FocusClear (a special reagent) [20, 21]. In such cases, the attenuation of light intensity due to photo scattering and absorption along the $\mathrm{z}$ axis is minute; therefore, a laser, which consumes less than $50 \%$ of the power required for imaging untransparent samples, is sufficient to activate the fluorescent molecules in samples. Moreover, the overall bleaching effect is also reduced because the amount of power required to activate a laser is very low.

The second solution modifies the microscope optics as an image captured $[22,23]$; and the third uses post-processing methods. For example, a computational method was proposed in [24] to extend the depth-of-field of images obtained by using conventional bright field microscopy. The scattering and absorption of fluorescence, which cause intensity loss mainly along the optical axis, and repeated scanning of an image are modeled as exponential functions of time and depth respectively [25]. The parameters of the functions are estimated and then used to compensate for the loss of intensity in an image. In some situations, researchers use bi-exponential functions to 
model the photobleaching effect $[26,27]$. Another class of methods matches the histograms of image stacks $[28,29]$. Other robust estimation methods have been proposed to remove outliers and adapt structures to compensate for the loss of intensity in microscope images [30].

We use FocusClear to obtain the confocal microscope images used in our experiments. Although the FocusClear technique is not yet popular, the high resolution and high quality confocal images that it yields have been used in several outstanding biomedical studies [31, 32, 33]. The bleaching along the z-axis is minimal; however, this is not the case along the border of overlapping intersections, so the intensity difference caused by bleaching becomes apparent in the same section, as shown in Figure 1. To resolve the problem, the proposed method blends asymmetrically informative image pairs by estimating a set of optimal blending vectors. In a sense, the solution compensates for the less-informative region by exploiting the information contained in the earlier-acquired image.

The remainder of this paper is organized as follows. In Section 2, we formulate the proposed approach as a constrained quadratic optimization problem; and in Section 3, we compare our experiment results with those of other methods. Then, we summarize our conclusions and consider future research directions in Section 4.

\section{Multiresolution Blending Coefficients}

We formulate our approach as a multiresolution parameter estimation problem, and propose an optimal parameter estimation procedure to derive an array of blending vectors $\mathrm{M}$. The vectors are used to compute the weighted average of two overlapping images on subbands of the image's pyramid rep- 
resentation. For an earlier-acquired image, $\mathcal{I}$, and a later-acquired image, $\mathcal{J}$, the blending procedure can be formulated as minimizing the function defined by

$$
\min _{\mathrm{M}} \mathcal{T}_{\text {blend }}(\mathrm{M} \mid \mathcal{I}, \mathcal{J})+\mu \mathcal{T}_{M}(\mathrm{M})
$$

where $\mathcal{T}_{\text {blend }}$ and $\mathcal{T}_{M}$ are the blending function term and the regularization term respectively, and the weighting $\mu$ balances the relative importance of the two terms. $\mathcal{T}_{\text {blend }}(\mathrm{M} \mid \mathcal{I}, \mathcal{J})$ denotes the cost of using $\mathrm{M}$ to blend images $\mathcal{I}$ and $\mathcal{J}$; and, $\mathcal{T}_{M}(\mathrm{M})$ measures the penalty of $\mathrm{M}$. The function $\mathcal{T}_{\text {blend }}$ requires that the combined image should be smooth and that it retains as much of the original information as possible. Specifically, it requires that the overlapping region is smooth and that the blending process does not affect the truthfulness of the area. Because adjacent rows of to-be-combined images tend to have similar image structures, the function $\mathcal{T}_{M}$ requires that the blending coefficients in adjacent rows are similar to each other.

The information in the overlapping regions of a pair of to-be-combined images is asymmetric; therefore, we have to find the optimal blending vectors as well as a suitable blending position to satisfy the above requirement.

\subsection{Selecting the blending position}

In fluorescence microscope imagery, the later-acquired image contains less trustworthy overlap information than the earlier-acquired image; thus, the blending position should be selected so that the information in the earlieracquired image is affected as little as possible by the blending process. Meanwhile, the weighting coefficients used to blend two overlapping images should be content dependent so that the values of the pixels in the transition zone can be evaluated according to the relative importance of two overlapping re- 
gions. However, because the complexity of deriving the blending coefficient for each pixel would be high, we simplify the procedure by assuming that all the pixels in a row are blended by the same vector.

Figure 4 illustrates the relationship between the blending position, the overlapping regions, and the transition region, which is the area where pixels are affected by the blending process. Let $u_{1}, u_{2}, \cdots, u_{n}$ be the pixels in the overlapping region of the earlier-acquired image $\mathcal{I}$, and let $v_{1}, v_{2}, \cdots, v_{n}$ be the corresponding pixels in the later-acquired image $\mathcal{J}$. When we blend confocal microscope images, the objective is to retain as much information as possible in the overlapping area of the earlier-acquired image $\mathcal{I}$; thus, we select the blending position that aligns with the right boundary of $\mathcal{I}$. Then, we can formulate Equation (1) precisely and estimate the blending parameters by the following optimization approach.

\subsection{Estimating optimal blending parameters}

In this subsection, we describe the blending function $\mathcal{T}_{\text {blend }}$, the penalty function $\mathcal{T}_{M}$, and the proposed constrained quadratic optimization algorithm used to estimate the blending parameters that solve Equation (1) optimally.

\subsubsection{The blending function}

We use the following blending function

$$
\mathcal{T}_{\text {blend }}(\mathrm{M} \mid \mathcal{I}, \mathcal{J})=\left\|\left[\left.\mathcal{I}\right|^{\mathrm{M}} \mathcal{J}\right]-[\mathcal{I} \mid \mathcal{J}]\right\|_{F}^{2}+\lambda\left\|\nabla_{h}\left[\left.\mathcal{I}\right|^{\mathrm{M}} \mathcal{J}\right]\right\|_{F}^{2}
$$

where $\left[\left.\mathcal{I}\right|^{\mathrm{M}} \mathcal{J}\right]$ denotes a two-dimensional matrix whose entries are the pixel values of the mosaiced image obtained by combining images $\mathcal{I}$ and $\mathcal{J}$ with the 
blending vectors $\mathrm{M}$; and $[\mathcal{I} \mid \mathcal{J}]$ denotes the matrix of the directly mosaiced image obtained by overlaying $\mathcal{I}$ on $\mathcal{J}$ without blending (i.e., the intensities of the pixels in the overlapping region of the directly mosaiced image are copied from the corresponding region of the earlier-acquired image $\mathcal{I}$ ), as shown in the rightmost image in Figure 1. Moreover, $\left\|\left[\left.\mathcal{I}\right|^{\mathrm{M}} \mathcal{J}\right]-[\mathcal{I} \mid \mathcal{J}]\right\|_{F}^{2}$ calculates the square of the Frobenius norm of the difference between the matrix of the blended image and that of the directly mosaiced image.

$\left\|\nabla_{h}\left[\left.\mathcal{I}\right|^{\mathrm{M}} \mathcal{J}\right]\right\|_{F}^{2}$ measures the smoothness of the blended image over the overlapping region of the two images; $\nabla_{h}$ is the first-order difference of adjacent pixels in a row; and the parameter $\lambda$ balances the importance of the integrity and smoothness terms.

The proposed blending process is applied on a Laplacian pyramid domain. Let us decompose each of the two to-be-combined images into $N$ levels. Because the images $\mathcal{I}$ and $\mathcal{J}$ are already registered, we can assume that they are combined row-by-row. Thus, different rows may yield different blending parameters.

$$
\begin{aligned}
& L_{N, r}=\left[\begin{array}{c}
\overline{\mathcal{I}^{[N]}(i)} \\
m_{1}^{r} \mathcal{I}^{[N]}\left(i_{1}\right)+\left(1-m_{1}^{r}\right) \mathcal{J}^{[N]}\left(j_{1}\right) \\
m_{2}^{r} \mathcal{I}^{[N]}\left(i_{2}\right)+\left(1-m_{2}^{r}\right) \mathcal{J}^{[N]}\left(j_{2}\right) \\
m_{3}^{r} \mathcal{I}^{[N]}\left(i_{3}\right)+\left(1-m_{3}^{r}\right) \mathcal{J}^{[N]}\left(j_{3}\right) \\
\frac{\mathcal{J}^{[N]}(j)}{}
\end{array}\right] \\
& =\left[\begin{array}{ccc}
\overline{0} & \overline{0} & \overline{0} \\
\mathcal{I}^{[N]}\left(i_{1}\right)-\mathcal{J}^{[N]}\left(j_{1}\right) & 0 & 0 \\
0 & \mathcal{I}^{[N]}\left(i_{2}\right)-\mathcal{J}^{[N]}\left(j_{2}\right) & 0 \\
0 & 0 & \mathcal{I}^{[N]}\left(i_{3}\right)-\mathcal{J}^{[N]}\left(j_{3}\right) \\
\overline{0} & \overline{0} & \overline{0}
\end{array}\right]\left[\begin{array}{c}
m_{1}^{r} \\
m_{2}^{r} \\
m_{3}^{r}
\end{array}\right]+\left[\begin{array}{c}
\overline{\mathcal{I}^{[N]}(i)} \\
\mathcal{J}^{[N]}\left(j_{1}\right) \\
\mathcal{J}^{[N]}\left(j_{2}\right) \\
\frac{\mathcal{J}^{[N]}\left(j_{3}\right)}{\mathcal{J}^{[N]}(j)}
\end{array}\right] \\
& =A_{N} m^{r}+C_{N} \text {, }
\end{aligned}
$$




\section{A. Blending a row:}

For simplicity, we assume that the blending coefficients in the same row in different Laplacian pyramid levels are the same. Without loss of generality, we use a blending vector of length 3: $\left[\begin{array}{lll}m_{1}^{r} & m_{2}^{r} & m_{3}^{r}\end{array}\right]^{T}$ for row $r$, where the superscript $T$ denotes the matrix transpose. Let the pixels in the blending zone of this row at the coarsest level, i.e., the $N^{\text {th }}$ level, of the Laplacian pyramids of images $\mathcal{I}$ and $\mathcal{J}$ be $i_{1}, i_{2}, i_{3}$ and $j_{1}, j_{2}, j_{3}$ respectively. The two rows are blended to obtain $m_{k}^{r} \mathcal{I}\left(r, i_{k}\right)+\left(1-m_{k}^{r}\right) \mathcal{J}\left(r, j_{k}\right)$, with $k=1,2,3$ in row $r$ of the coarsest level of the blended image, as shown in Figure 5. Note that the intersection of pixels $i_{3}$ and $j_{3}$ is chosen as the blending position. The mosaic result of the $N^{\text {th }}$ level Laplacian subband of this row can be represented by Equation (3), where $\overline{\mathcal{I}^{[N]}(i)}$ and $\overline{\mathcal{J}^{[N]}(j)}$ denote, respectively, the pixels not affected by blending the $N^{\text {th }}$ level $\mathcal{I}$ and $\mathcal{J}$ Laplacian images; and $\overline{0}$ denotes a sequence of zeros of an appropriate length. The mosaiced Laplacian subbands of this row at other levels can be derived in the same way.

The width of the transition area depends on the number of pyramid decompositions, $N$, and the length of the filter, $l$, used to construct the pyramid. Let $v$ be the length of the blending vector. Then, the width of the transition region, $W_{\text {trans }}$, can be calculated as follows:

$$
W_{\text {trans }}(N)=2 W_{\text {trans }}(N-1)+l-2 \text {, with } W_{\text {trans }}(0)=v \text {. }
$$

Generally speaking, a wider transition area yields a smoother blending result, but it preserves less original information. The approach that sets the blending position on the boundary of $\mathcal{I}$ yields a smooth blended image that preserves as much information as possible in the earlier-acquired image $\mathcal{I}$. 
As shown in Figure 3, the Gaussian pyramid at the $(N-1)^{\text {th }}$ level of the combined image can be obtained by performing an expand operation on $L_{N, r}$ and adding the result to $L_{N-1, r}$. Let $U_{k}$ and $W_{k}$ denote, respectively, the upsampling kernel and the low-pass kernel at the $k^{t h}$ level. To obtain the combined image at the $(N-1)^{t h}$ level of the Gaussian pyramid, the expand operation up-samples $L_{N, r}$ by applying $U_{N}$ and smoothes the result by applying $W_{N}$, which yields

$$
\begin{aligned}
G_{N-1, r} & =L_{N-1, r}+W_{N} U_{N} L_{N, r} \\
& =\left(A_{N-1}+W_{N} U_{N} A_{N}\right) m^{r}+\left(C_{N-1}+W_{N} U_{N} C_{N}\right) .
\end{aligned}
$$

Based on the above procedure, the mosaic result at the finest resolution of the Gaussian pyramid can be derived by

$$
G_{0, r}=\mathrm{A}^{r} m^{r}+\mathrm{C}^{r}
$$

where

$$
\mathrm{A}^{r}=A_{1}+\sum_{j=2}^{N}\left(\prod_{i=2}^{j} W_{i} U_{i}\right) A_{j}
$$

and

$$
\mathrm{C}^{r}=C_{1}+\sum_{j=2}^{N}\left(\prod_{i=2}^{j} W_{i} U_{i}\right) C_{j}
$$

Let $[\mathcal{I} \mid \mathcal{J}]^{r}$ denote the signal obtained by combining the $r$-th rows of images $\mathcal{I}$ and $\mathcal{J}$ directly. According to Equation (2), the cost of blending row $r$ with 
vector $m^{r}$ is

$$
\left\|\left(\mathrm{A}^{r} m^{r}+\mathrm{C}^{r}\right)-[\mathcal{I} \mid \mathcal{J}]^{r}\right\|_{2}^{2}+\lambda\left\|D\left(\mathrm{~A}^{r} m^{r}+\mathrm{C}^{r}\right)\right\|_{2}^{2},
$$

where $D$ is the kernel matrix of the first-order difference along the direction of the column. Let

$$
\mathrm{P}^{r}=[\mathcal{I} \mid \mathcal{J}]^{r}-\mathrm{C}^{r} .
$$

Equation (9) can now be re-written as

$$
\left\|\mathrm{A}^{r} m^{r}-\mathrm{P}^{r}\right\|_{2}^{2}+\lambda\left\|D\left(\mathrm{~A}^{r} m^{r}+\mathrm{C}^{r}\right)\right\|_{2}^{2} .
$$

Note that the Frobenius norm of a 1-D vector is identical to the vector's 2-norm.

B. Blending all the rows in the overlapping region:

Before describing how an image pair is mosaiced by blending all $K$ rows 
in the overlapping area, we define the following symbols:

$$
\begin{aligned}
& \mathbf{A}=\left[\begin{array}{cccc}
\mathrm{A}^{1} & 0 & \cdots & 0 \\
0 & \mathrm{~A}^{2} & \cdots & 0 \\
\vdots & & \ddots & \\
0 & 0 & \cdots & \mathrm{A}^{K}
\end{array}\right] \\
& \mathbf{D A}=\left[\begin{array}{cccc}
D A^{1} & 0 & \cdots & 0 \\
0 & D A^{2} & \cdots & 0 \\
\vdots & & \ddots & \\
0 & 0 & \cdots & D A^{K}
\end{array}\right] \text {, } \\
& \mathbf{P}=\left[\begin{array}{llll}
\mathrm{P}^{1} & \mathrm{P}^{2} & \cdots & \mathrm{P}^{\mathrm{K}}
\end{array}\right]^{T}, \\
& \mathbf{C}=\left[\begin{array}{llll}
\mathrm{C}^{1} & \mathrm{C}^{2} & \cdots & \mathrm{C}^{\mathrm{K}}
\end{array}\right]^{T} \text {, and } \\
& \mathrm{DC}=\left[\begin{array}{llll}
D \mathrm{C}^{1} & D \mathrm{C}^{2} & \cdots & D \mathrm{C}^{\mathrm{K}}
\end{array}\right]^{T} \text {, }
\end{aligned}
$$

where the definitions of $\mathrm{A}^{k}, \mathrm{C}^{k}$ and $\mathrm{P}^{k}$ are the same as in Equations (7), (8) and (10) respectively, and the superscript $k$ denotes the row-index; $D$ is the kernel matrix of the first order difference along the direction of the column; and the superscript $T$ denotes a transpose operation. The block diagonal matrices $\mathbf{A}$ and $\mathbf{D A}$ are introduced to extend the formulation of blending a row to blending sets of rows. Accordingly, the blending function for mosaicing the overlapping region can be written as follows:

$$
\begin{aligned}
\mathcal{T}_{\text {blend }}(\mathrm{M} \mid \mathcal{I}, \mathcal{J}) & =\left\|\left[\left.\mathcal{I}\right|^{\mathrm{M}} \mathcal{J}\right]-[\mathcal{I} \mid \mathcal{J}]\right\|_{F}^{2}+\lambda\left\|\nabla\left[\left.\mathcal{I}\right|^{\mathrm{M}} \mathcal{J}\right]\right\|_{F}^{2} \\
& =\|\mathbf{A M}-\mathbf{P}\|_{2}^{2}+\lambda\|\mathbf{D A M}+\mathbf{D C}\|_{2}^{2}
\end{aligned}
$$

where $\mathrm{M}$ is the vector formed by cascading the blending vector of each row. 
The vector is defined as

$$
\mathrm{M}=\left[\begin{array}{lllllll}
m_{1}^{1} & m_{2}^{1} & m_{3}^{1} & \ldots & m_{1}^{K} & m_{2}^{K} & m_{3}^{K}
\end{array}\right]^{T} .
$$

\subsubsection{The penalty function of the blending vector}

Because adjacent rows of images tend to have similar structures and intensities, the function $\mathcal{T}_{M}$ measures the discrepancy between the blending coefficients in adjacent rows. Let

$$
\mathbf{M}=\left[\begin{array}{cccc}
m_{1}^{1} & m_{1}^{2} & \cdots & m_{1}^{K} \\
m_{2}^{1} & m_{2}^{2} & \cdots & m_{2}^{K} \\
m_{3}^{1} & m_{3}^{2} & \cdots & m_{3}^{K}
\end{array}\right]^{T}
$$

Note that $\mathrm{M}=\operatorname{vec}\left(\mathbf{M}^{T}\right)$, where vec is the vec operator in matrix calculus. The penalty function can be defined as the sum of the squared differences of the blending vectors of two adjacent rows:

$$
\begin{aligned}
\mathcal{T}_{M}(\mathrm{M}) & =\left\|\nabla_{y} \mathbf{M}\right\|_{F}^{2} \\
& =\sum_{j=1}^{K-1} \sum_{i=1}^{3}\left(m_{i}^{j+1}-m_{i}^{j}\right)^{2}
\end{aligned}
$$

where $\nabla_{y}$ is the derivative along the direction of the column. The objective of the penalty is to ensure that the values of adjacent blending vectors are 
similar. Equation (16) can be re-written in the following equivalent form:

$$
\begin{aligned}
\mathcal{T}_{M}(\mathrm{M})= & \left\|\left[\begin{array}{ccccccc}
-1 & 0 & 0 & 1 & 0 & 0 & \ldots \\
0 & -1 & 0 & 0 & 1 & 0 & \ldots \\
0 & 0 & -1 & 0 & 0 & 1 & \ldots \\
\vdots & & & \ddots & & & \\
\vdots & & & & \ddots &
\end{array}\right] \quad \mathrm{M}\right\|_{F}^{2} \\
& =\|\mathbf{H M}\|_{2}^{2},
\end{aligned}
$$

where $\mathbf{H M}$ is a vector, and $\mathbf{H}$ is a Toeplitz matrix denoting the discrete convolution.

\subsubsection{Solving the optimal blending parameters}

Combining the results of Equations (13) and (17), the objective function in Equation (1) of the blending procedure can be derived as follows:

$$
\begin{aligned}
\mathcal{T}_{\text {blend }}(\mathrm{M} \mid \mathcal{I}, \mathcal{J})+\mu \mathcal{T}_{M}(\mathrm{M}) \\
=\|\mathbf{A M}-\mathbf{P}\|_{2}^{2}+\lambda\|(\mathbf{D A M}+\mathbf{D C})\|_{2}^{2}+\mu\|\mathbf{H} \mathbf{M}\|_{2}^{2} \\
=\mathrm{M}^{T}\left(\mathbf{A}^{T} \mathbf{A}+\lambda \mathbf{D} \mathbf{A}^{T} \mathbf{D} \mathbf{A}+\mu \mathbf{H}^{T} \mathbf{H}\right) \mathrm{M} \\
-2\left(\mathbf{P}^{T} \mathbf{A}-\lambda \mathbf{D} \mathbf{C}^{T} \mathbf{D} \mathbf{A}\right) \mathrm{M}+\mathbf{P}^{T} \mathbf{P}+\mathbf{D} \mathbf{C}^{T} \mathbf{D C} .
\end{aligned}
$$

By omitting the constant terms and letting $\mathrm{Q}=\mathbf{A}^{T} \mathbf{A}+\lambda \mathbf{D} \mathbf{A}^{T} \mathbf{D A}+\mu \mathbf{H}^{T} \mathbf{H}$ and $\mathrm{f}^{T}=-\left(\mathbf{P}^{T} \mathbf{A}-\lambda \mathbf{D} \mathbf{C}^{T} \mathbf{D A}\right)$, the objective function becomes the following quadratic programming problem:

$$
\min _{\mathrm{M}} \mathcal{T}_{\text {blend }}(\mathrm{M} \mid \mathcal{I}, \mathcal{J})+\mu \mathcal{T}_{M}(\mathrm{M})=\min _{\mathrm{M}} \frac{1}{2} \mathrm{M}^{T} \mathrm{QM}+\mathrm{f}^{T} \mathrm{M}
$$


Recall that, because of the photobleaching of microscope images, the overlapping region of the earlier-acquired image is more informative than that of the later-acquired image. To preserve as many Laplacian coefficients as possible during the blending of the earlier-acquired image, we require that the blending coefficients for that image should be larger than those for the later-acquired image. The requirement corresponds to the constraint that each element in $\mathrm{M}$ is not less than $\mathrm{M}_{\text {min }}$, whose value is set at 0.5 in our experiments. We also require that the intensity of the mosaic image is upper bounded, which corresponds to the constraint that each element in $\mathrm{M}$ is not larger than $\mathrm{M}_{\max }$, whose value is set at 1 in our experiments. In [17], the coefficients of the mosaic image form a convex combination of the corresponding coefficients in the earlier- and later-acquired images. This can also be formulated easily as a linear constraint and incorporated into our framework.

\section{$3 \quad$ Experiment Results}

In our implementation, we use the Daubechies bi-orthogonal 5-3 low-pass filter to decompose and reconstruct an image pyramid [34]. Specifically, we use the 5-tap analysis low-pass filter in the reduce operation and the 3-tap synthesis low-pass filter in the expand operation. The length of a row's blending vector is 3 . Because we assume that the image pair is already registered, the overlapping region of each pair of images is determined by the registration process. In all the experiments described below, we decompose the images into 5 levels; hence, according to Equation (4), the width of the transition region is 127 pixels, which is $\frac{1}{8}$ of the width of image $\mathcal{I}$. The parameters $\lambda$ and $\mu$ in Equation (18) are set at 12 and 5 respectively in all 
cases. The values of the two parameters are determined experimentally. The optimal blending vectors $M$ are derived by solving the constrained quadratic problem in Equation (18) with the constraint that each element of $M$ belongs to the interval $[0.5,1]$. There are several outstanding methods that can derive the optimal solution of the constrained quadratic problem $[35,36,37]$. We use Matlab's built-in functions, quadprog.m or qpdantz.m, to solve the equation. Note that qpdantz.m implements Dantzig-Wolfe's algorithm [38]. Further, details can be found in the Chapter 7 of [39].

The images were acquired by using a Zeiss LSM 510 confocal microscope with a 40x C-Apochromat water-immersion objective lens. The fluorochrome can be green fluorescent protein (GFP) or a fluorescent antibody. The procedures for preparing and imaging the samples are exactly the same as those described in [33] in the sections entitled "Sample Preparation" and "Confocal Imaging." The images to be blended are registered manually. In the following, we evaluate the proposed algorithm's performance in combining confocal microscope images.

Figure 6 shows a sequence of six images taken from fluorescent confocal microscope images of a Drosophila's brain. The images were captured in a clockwise direction, starting from the top left-hand image. Although some images overlap with one or more other images, we only combined two images at a time. The pairs (OPi,OPi') with $i=1, \cdots, 7$ are the overlapping areas of the images. The image containing OPi was taken before that containing $\mathrm{OPi}$ '. In all cases, the intensity of $\mathrm{OP} i$ is larger than that of $\mathrm{OP} i$ ' except in OP7 and OP7'. Biologists adjusted the intensity of the bottom left-hand image manually by multiplying a constant gain on the whole image. Since the bottom-left image has two overlapping areas, $\mathrm{OP} 7^{\prime}$ and $\mathrm{Op} 6^{\prime}$, it is very 
difficult to adjust the image correctly; hence, the later-acquired area OP7' appears to be lighter than OP7. This shows that compensating for intensityloss by adjusting the mean values is not a straightforward task. To obtain the whole mosaic image, we mosaic the top images to form one image, and do the same for the bottom images. Then, the two resulting images are combined. To obtain the top mosaic image, we follow the blending line of images (a) and (b) on the right-hand boundary of OP1' and the blending line of images (b) and (c) on the right-hand boundary of OP2'. Similarly, to obtain the bottom mosaic image, we follow the blending line of images (d) and (e) on the left-hand boundary of OP6' and that of images (e) and (f) on the left-hand boundary of OP4'. When the resulting top and bottom mosaic images are combined, the blending lines between the upper-mosaiced and the lower-mosaiced half images are on the bottom boundaries of OP7', OP5 ', and Op3' respectively.

Figure 7 compares the results of mosaicing the component images in Figure 6 by the proposed method (top), Burt and Adelson's method (middle), and MosaicJ (bottom). The blending coefficients used to obtain the middle image are $0.25,0.5$, and 0.75 . For example, let $i_{1}, i_{2}, i_{3}$ and $j_{1}, j_{2}, j_{3}$ be, respectively, the coefficients of the over-lapping areas of two lines in the $l^{\text {th }}$-level Laplacian images. Then, the blended values are $0.75 i_{1}+0.25 j_{1}, 0.5 i_{2}+0.5 j_{2}$ and $0.25 i_{3}+0.75 j_{3}$. Our method yields better contrast as shown by the rectangular area in the overlapping regions; however, MosaicJ yields a smoother result, e.g., the ellipse region of the bottom image is more blurred than the regions in the other two images. Note that the blending process only affects the regions in the transition zone. We observe that the three methods derive similar results in non-overlapping regions. Figure 8 shows some mosaicing 
results of Drosophila brain images at different depths. Note that when we process the EM images, we set $\mathrm{M}_{\max }=1$ and $\mathrm{M}_{\min }=0$ because they are equally informative.

The other set of data contains mouse pancreas images that were also acquired by a Zeiss LSM 510 confocal microscope under almost the same conditions as those described earlier. The top row in Figure 9 shows the images to be combined, and the bottom image is our mosaic image. Since the right-hand image was acquired later, the blending position, indicated by the arrow in the middle image of Figure 9, is selected near the right-hand boundary of the overlapping area of the top left image. These images only contain two channels: an R-channel for nuclei and a G-channel for other tissues. Due to possible vibrations, there was a misalignment in the slice depth when these two image stacks were acquired; thus, the size of the same tissues in the top-left and top-right images may look slightly different. Our algorithm mosaiced the R- and G- channels separately; that is, the blending vectors for the channels were estimated independently. For example, the optimized blending vectors of the $994^{t h}, 995^{t h}$ and $996^{t h}$ rows for the R-channel are $\left[\begin{array}{llll}0.9500 & 0.9500 & 0.9500\end{array}\right]^{T},\left[\begin{array}{llll}0.9500 & 0.9500 & 0.9500\end{array}\right]^{T}$ and $\left[\begin{array}{lll}0.9472 & 0.9472 & 0.9472\end{array}\right]^{T}$ respectively; and those for the G-channel are $[0.49380 .95000 .5474]^{T}$, $\left[\begin{array}{llll}0.7322 & 0.9500 & 0.7464\end{array}\right]^{T}$ and $\left[\begin{array}{lll}0.8691 & 0.9472 & 0.8672\end{array}\right]^{T}$ respectively. We observe that these two sets of blending coefficients vary gradually row-by-row due to the regularization term in Equation (1). For regions that do not contain any nuclei, the corresponding blending coefficients for the R-channel remain unchanged, but those for the G-channel vary.

Finally, applying our algorithm on all the images, we measured the average computational time versus the number of rows. The results are shown 
in Figure 10. The data was processed on a Windows 7 server with an Intel i7 $\mathrm{CPU}(3.07 \mathrm{GHz})$ and $6 \mathrm{~GB}$ RAM; and the algorithm was implemented by Matlab R2009a without parallel computing. We observe that the average time required for mosaicing is almost constant when the number of rows is less than 640 , but it increases thereafter. To reduce the average computation time, we can either downsample the rows or partition the images. By downsampling the rows, we can obtain images with fewer rows. After deriving the optimal blending coefficients for the rows of the downsampled images, we can interpolate the blending coefficients for rows that were not sampled in the downsampling process. By partitioning the images, we can obtain several sub-images with fewer rows. The sub-images are processed independently and their results are combined to obtain a final mosaic image.

\section{Conclusion}

Mosaicing of confocal microscope images has some unique and latent properties that do not appear in other applications. For example, because of the photobleaching effect, earlier-acquired images should be given more weight than later-acquired images in the mosaicing process. We incorporate these properties into a mosaicing procedure and define a multi-resolution optimum blending parameter estimation problem that can be solved by quadratic programming with linear constraints. The perceptual quality of the resulting mosaic images is compared with that of the results derived by Burt and Adelson's algorithm and the MosaicJ algorithm. Based on the proposed optimization framework, it would be easy to extend the proposed method to other scenarios with case-dependent constraints.

Finally, in our future research, we will consider developing a technique 
that can combine multiple image stacks simultaneously as well as a standard perceptual measurement of the mosaic results for comparing different methods.

\section{Acknowledgments}

The authors wish to thank Professor Ann-Shyn Chiang, the Program Director of the Brain Research Center at National Tsing Hua University, as well as Professor Shiue-Cheng Tang and Dr. Hsiu-Ming Chang for their enthusiastic support and for providing the confocal microscope images.

\section{References}

[1] X. Zhou and S. T. C. Wong, "Informatics challenges of high-throughput microscopy", IEEE Signal Proc. Mag., vol. 23, no. 3, pp. 63-72, 2006.

[2] C. Vonesch, F. Aguet, J. K. Vonesch, and M. Unser, "The colored revolution of bioimaging", IEEE Signal Proc. Mag., vol. 23, no. 3, pp. 20-31, 2006.

[3] H. Y. Shum and R. Szeliski, "System and experiment paper: construction of panoramic image mosaics with global and local alighment", Int. J. Comput. Vis., vol. 36, no. 2, pp. 101-130, 2000.

[4] S. E. Chen, "QuickTime VR - an image-based approach to virtual environment navigation", SIGGRAPH, 1995, pp. 29-38. 
[5] J. Jia and C.-K. Tang, "Eliminating structure and intensity misalignment in image stitching", Proc. The Tenth IEEE International Conference on Computer Vision (ICCV2005), 2005, pp. 1651-1658.

[6] A. Zomet, A. Levin, S. Peleg, and Y. Weiss, "Seamless image stitching by minimizing false edges", IEEE Trans. Image Process., vol. 15, no. 8, pp. 969-977, 2005.

[7] M. Brown and D. G. Lowe, "Automatic panoramic image stitching using invariant features", Int. J. Comput. Vision, vol. 74, no.1, pp.59-73, 2006.

[8] J. Kopf, M. Uyttendaele, O. Deussen, and M. F. Cohen, "Capturing and viewing gigapixel images", ACM Transactions on Graphics, vol. 26, no. $3,2007$.

[9] P. Pérez, M. Gangnet, and A. Blake, "Poisson Image Editing", ACM Transactions on Graphics, vol. 22, no. 3, pp. 313-318, 2003.

[10] P. Viola and W. M. Wells III, "Alignment by maximization of mutual information", Int. J. Comput. Vision, vol. 24, pp. 137-154, 1997.

[11] F. Maes, A. Collignon, D. Vandermeulen, G. Marchal, and P. Suetens, "Multimodality image registration by maximization of mutual information", IEEE Trans. Med. Imaging, vol. 16, no. 2, pp. 187-198, 1997.

[12] J. B. A. Maintz and M. A. Viegever, "A Survey of medical image registration", Med. Image Anal., vol. 2, no. 1, pp. 1-36, 1998.

[13] A. Gholipour, N. Kehtarnavaz, R. Briggs, M. Devous, and K. Gopinath, "Brain functional localization: A survey of image registration techniques", IEEE Trans. Med. Imaging, vol. 26, no. 4, pp. 427-451, 2007. 
[14] G. Yang, C. V. Stewart, M. Sofka, and C.-L. Tsai, "Registration of challenging image pairs: initialization, estimation, and decision", IEEE Trans. Pattern Anal. Mach. Intell., vol. 29, no. 11, pp. 1973-1989, 2007.

[15] A. Eden, M. Uyttendaele, and R. Szeliski, "Seamless image stitching of scenes with large motions and exposure differences", Proc. IEEE Conference on Computer Vision and Pattern Recognition (CVPR2006), 2006, pp. 2498-2505.

[16] P. Thévenaz and M. Unser, "User-friendly semiautomated assembly of accurate image mosaics in microscopy", Microscopy Research And Technique, vol. 70, no.2, pp. 135-146, 2007

[17] P. J. Burt and E. H. Adelson, " A multiresolution spline with application to image mosaics", ACM Transactions on Graphics, vol. 2, no. 4, pp. 217236, 1983.

[18] P. J. Burt and E. H. Adelson, "The Laplacian pyramid as a compact image code", IEEE Trans. Commun., vol. COM-31, pp. 532-540, 1983.

[19] M. S. Su, W. L. Hwang, and K. Y. Cheng, "Analysis on multiresolution mosaic images", IEEE Trans. Image Process., vol. 3, no. 7, pp. 952-959, 2004.

[20] Y. C. Liu, and A. S. Chiang, "High-resolution confocal imaging and three-dimensional rendering", Methods, vol. 30, pp. 86-93, 2003.

[21] A.-S. Chiang, "Aqueous Tissue Clearing Solution", US Patent 6472216, 2002.

[22] E. R. Dowski and W. T. Cathey, "Extended depth of field through wavefront coding", Appl. Opt., vol. 43, no. 11, pp. 1859-1866, 1995. 
[23] R. Ng, M. Levoy, M. Bredif, M. Horowitz, and P. Hanrahan, "Light field photography with a hand-held plenoptic camera", Stanford Univ. Computer Science Tech Report, CSTR 2005-02.

[24] F. Aguet, D. Van De Ville, and M. Unser, "Model-based 2.5-D deconvolution for extended depth of field in brightfield microscopy", IEEE Trans. Image Process., vol. 17, no. 7, pp. 1144-1153, 2008.

[25] J. Rigaut and J. Vassy, "High-resolution 3D images from confocal scanning laser microscopy: quantitative study and mathematical correction of the effects from bleaching and fluorescence attenuation in depth", Anal. Quant. Cytol., vol. 13, pp. 223-232, 1991.

[26] R. Ghauharali, J. Hofstraat, and G. Brakenhoff, "Fluorescence photobleaching-based shading correction for fluorescence microscopy", $J$. Microsc., vol. 192, pp. 99-113, 1998.

[27] J. Markham and J. Conchello, "Artefacts in restored images due to intensity loss in 3D fluorescence microscopy", J. Microsc., 204, pp. 93$98,2001$.

[28] S. Negahdaripour and C.-H. Yu, "A generalized brightness change model for comupting opticl flow", Proc. of Fourth International Conference on Comupter Vision, 1993, pp. 2-11.

[29] S. Gopinath, N. Thakoor, J. Gao, and K. Luby-Phelps, "A statistical approach for intensity loss compensation of confocal microscopy images", Proc. IEEE The Fourteenth International Conference on Image Processing (ICIP2007) 2007, pp. VI-249 - VI-252. 
[30] C. Kervrann, D. Legland, and L. Pardini, "Robust incremental compensation of the light attenuation with depth in 3d fluorescence microscopy", J. Microsc., vol. 214, no.3, pp. 297-314, 2004.

[31] H. H. Lin, Jason S. Y. Lai, A. L. Chin, Y. C. Chen, and A. S. Chiang, "A Map of Olfactory Representation in the Drosophila Mushroom Body", Cell, vol. 128, pp. 1205-1217, 2007.

[32] Y.-Y. Fu, C.-W. Lin, G. Enikolopov, E. Sibley, A.-S. Chiang, and S.C. Tang, "Microtome-Free 3-Dimensional Confocal Imaging Method for Visualization of Mouse Intestine with Subcellular-Level Resolution", Gastroenterology, vol. 137, issue 2, pp. 453-465, 2009.

[33] A. S. Chiang et. al., "Three-Dimensional Reconstruction of Brain-wide Wiring Networks in Drosophila at Single-Cell Resolution", Curr. Biol., vol. 21, issue 1, pp. 1-11, 2010.

[34] I. Daubechies, "Ten lectures no wavelets", SIAM, 1994.

[35] A. Ravindran, K. M. Ragsdell, and G. V. Reklaitis, "Engineering optimization methods and applications", 2nd Ed., WILEY, 2006.

[36] E. K. P. Chong and S. H. Żak, "An introduction to optimization", 2nd Ed., WILEY, 2001.

[37] T.F. Coleman and Y. Li, "A Reflective newton method for minimizing a quadratic function subject to bounds on some of the variables", SIAM J. Optimiz., vol. 6, no. 4, pp. 1040-1058, 1996.

[38] R. Fletcher, "Practical methods of optimization", John Wiley \& Sons, 1987. 
[39] G.B. Dantzig, "Linear programming and extensions", Princeton University Press, 1963. 


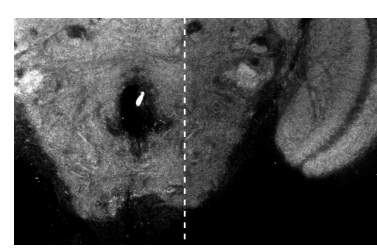

(a)

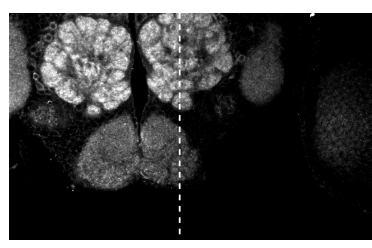

(d)

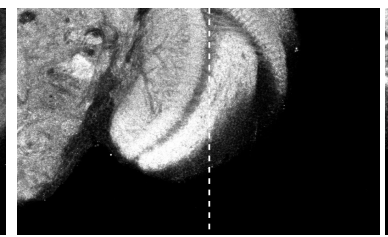

(b)

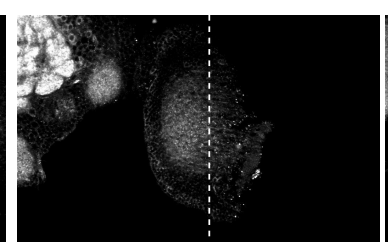

(e)

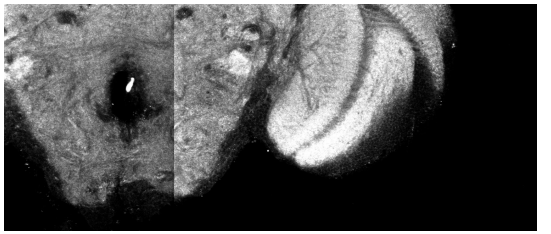

(c)

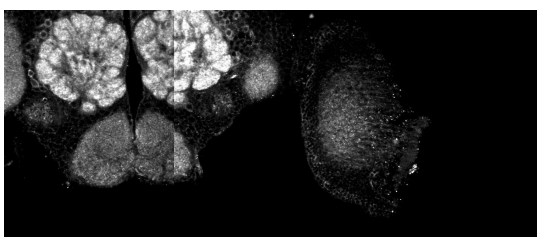

(f)

Figure 1: Images affected by photobleaching. The dashed-line indicates the boundary of the overlapping region. (a) and (d): the later-acquired image $\mathcal{J}$; (b) and (c): the earlier-acquired image $\mathcal{I}$. (c) and (f): the direct stitching result of $\mathcal{I}$ and $\mathcal{J}$, denoted as $[\mathcal{I} \mid \mathcal{J}]$ hearafter. The overlapping region of $\mathcal{J}$ is much darker than that of $\mathcal{I}$ because of repeated imaging. 


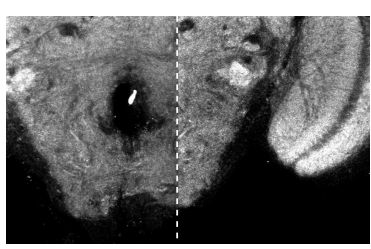

(a)

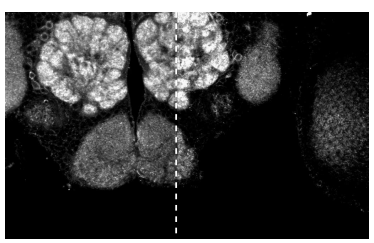

(c)

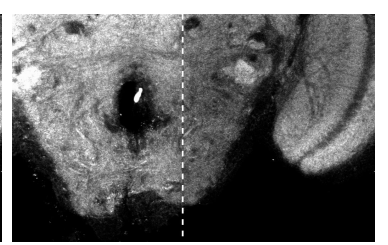

(b)

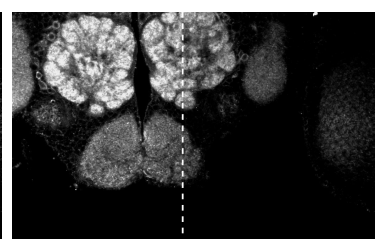

(d)

Figure 2: Adjusting the contrast of later-acquired images. The dashed-line indicates the boundary of the overlapping region. (a) and (c): the contrast of the overlapping regions of the images in Figure 1(a) and Figure 1(d) after adjustment. Note the discontinuity on the boundary of the overlapping regions in (a) and (c). (b) and (d): the contrast of the whole images in Figure 1(a) and Figure 1(d) after adjustment. Note that the contrast of the pixels to the left of the overlapping region in images (b) and (d) matches to that of the earlier-acquired images. However, the contrast of the pixels to the right of the overlapping regions in (b) and (d) is still lower. The contrast in images (a)-(d) is adjusted as follows. First, we obtain the histograms of the pixels in the overlapping regions of earlier-acquired images and in the to-be-adjusted regions of later-acquired images. Then, we remove the intensity below $1 \%$ and above $99 \%$ from all the histograms. Finally, we map the resultant histograms of the later-acquired image to those of the earlier-acquired image by linear stretching. 


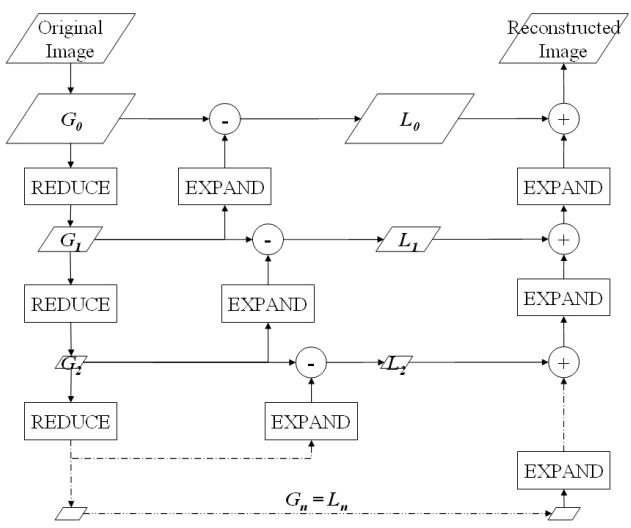

Figure 3: The Image Pyramid Structure. The Gaussian pyramid is $G_{0}, \cdots$, $G_{n}$, and the Laplacian pyramid is $L_{1}, \cdots, L_{N}$. The pyramid representation of the input image is a collection of pyramid sub-band images comprised of $G_{n}\left(=L_{n}\right)$ and $L_{n-1}, \cdots, L_{0}$. The sizes of the images are different. The smaller images correspond to lower frequency bands. As shown in the rightmost column, the original image, $G_{0}$, can always be recovered from the pyramid representation of the image.

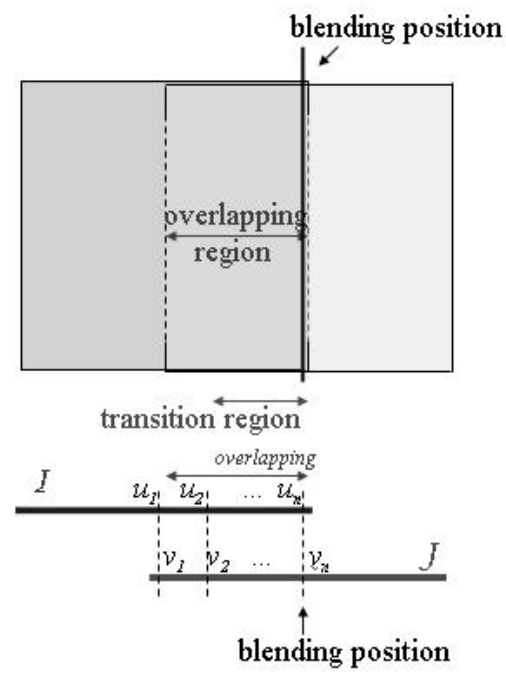

Figure 4: Top: The blending position, the overlapping region and the transition region. Image $\mathcal{I}$ is the earlier-acquired image and $\mathcal{J}$ is the later-acquired image. The width of the transition region depends on the levels of pyramid decomposition. Bottom: The blending position aligns the pixels $u_{n}$ in $\mathcal{I}$ and $v_{n}$ in $\mathcal{J}$. 
Figure 5: The blending coefficients are $\left[\begin{array}{ll}m_{1} & m_{2} \\ m_{3}\end{array}\right]^{T}$. The coefficients for the top line are $m_{1}, m_{2}, m_{3}$ and those for the bottom line are $1-m_{1}, 1-m_{2}$, and $1-m_{3}$. $\mathcal{I}^{[l]}$ and $\mathcal{J}^{[l]}$ denote the $l^{\text {th }}$ level Laplacian images of $\mathcal{I}$ and $\mathcal{J}$ respectively.

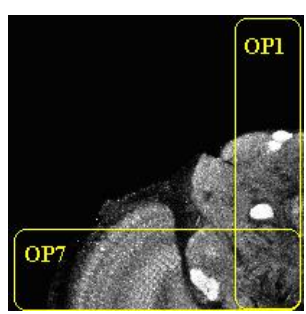

(a)

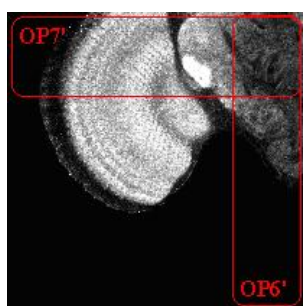

(d)

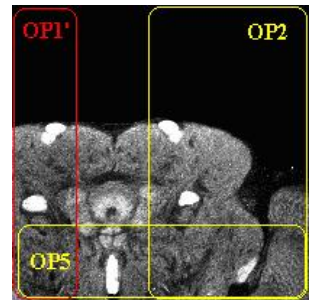

(b)

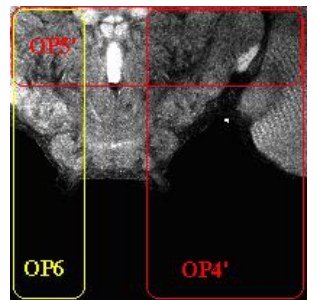

(e)

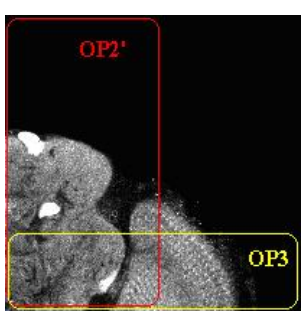

(c)

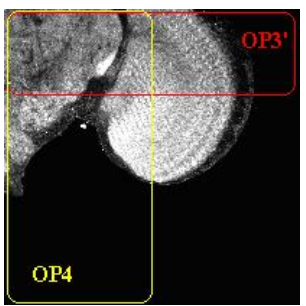

(f)

Figure 6: Confocal microscope images of a Drosophila's brain. The dimensions of each image stack are $1024 \times 1024 \times 123$, and the sampling resolutions along the $\mathrm{x}^{-}, \mathrm{y}$ - and z-directions are $0.32 \mu \mathrm{m}, 0.32 \mu \mathrm{m}$ and $1.0 \mu \mathrm{m}$ respectively. 

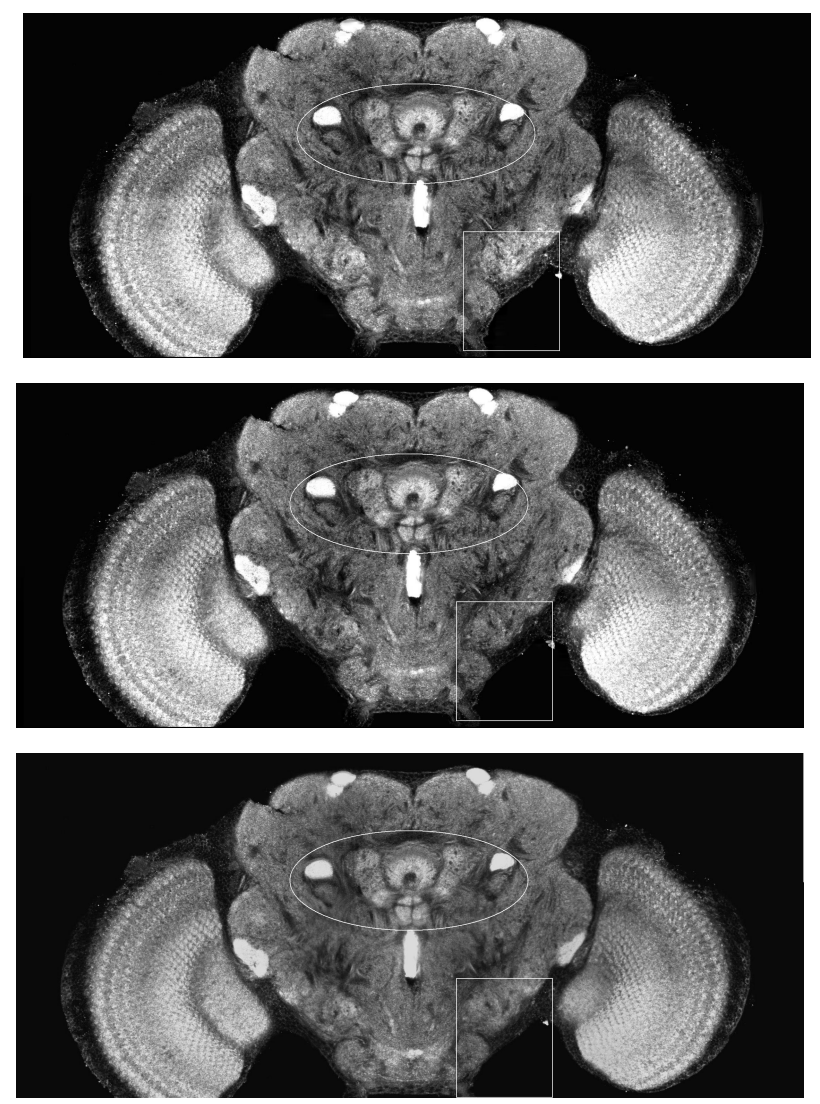

Figure 7: Comparison of mosaicing the images in Figure 6 by different methods. Top: the mosaic image obtained by the proposed method. Middle: the mosaic image derived by the method in [17]. Bottom: the mosaic image obtained by using the MosaicJ algorithm. The dimensions of the mosaiced result are $2272 \times 992$. Some parts of the images are highlighted to indicate where the contrast of the mosaiced image derived by our method is better than that obtained by other methods. 

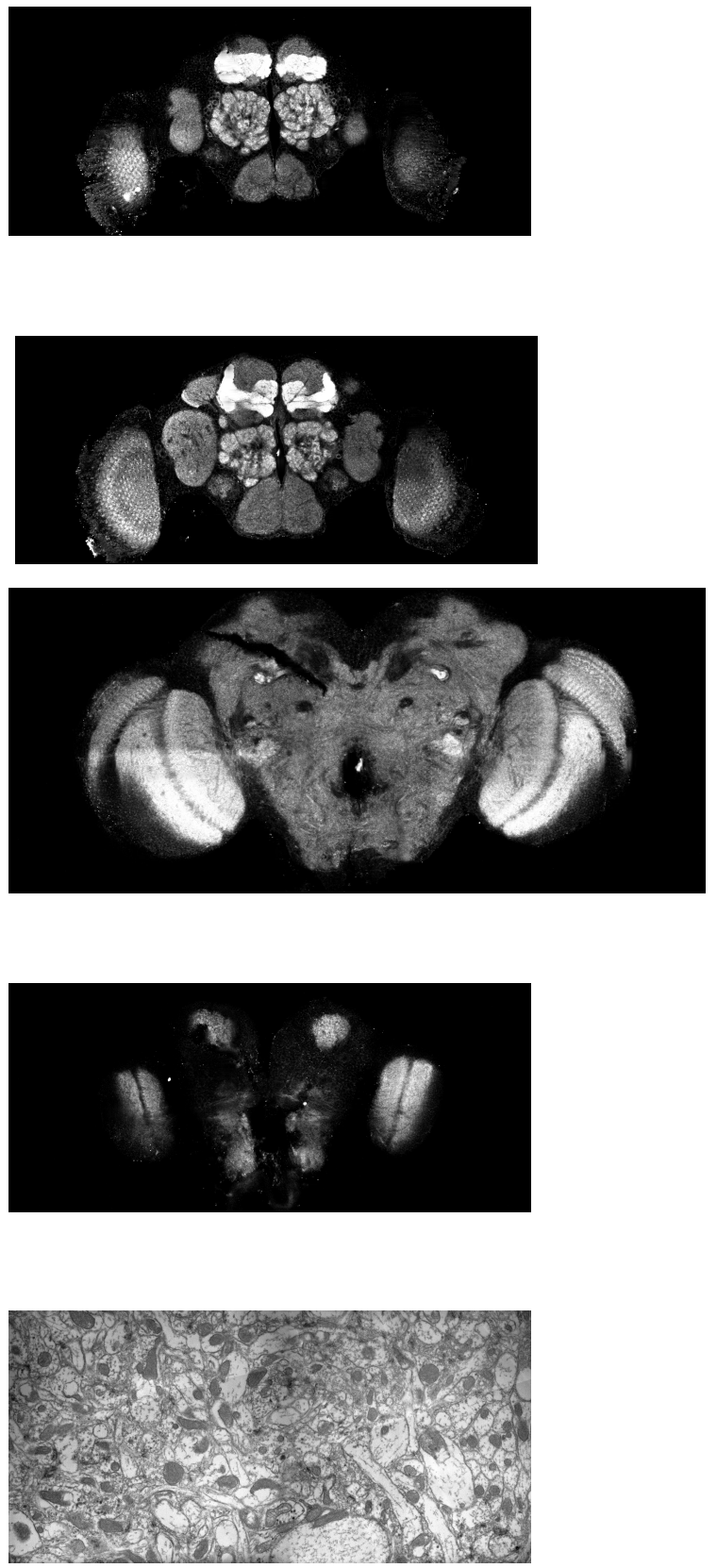
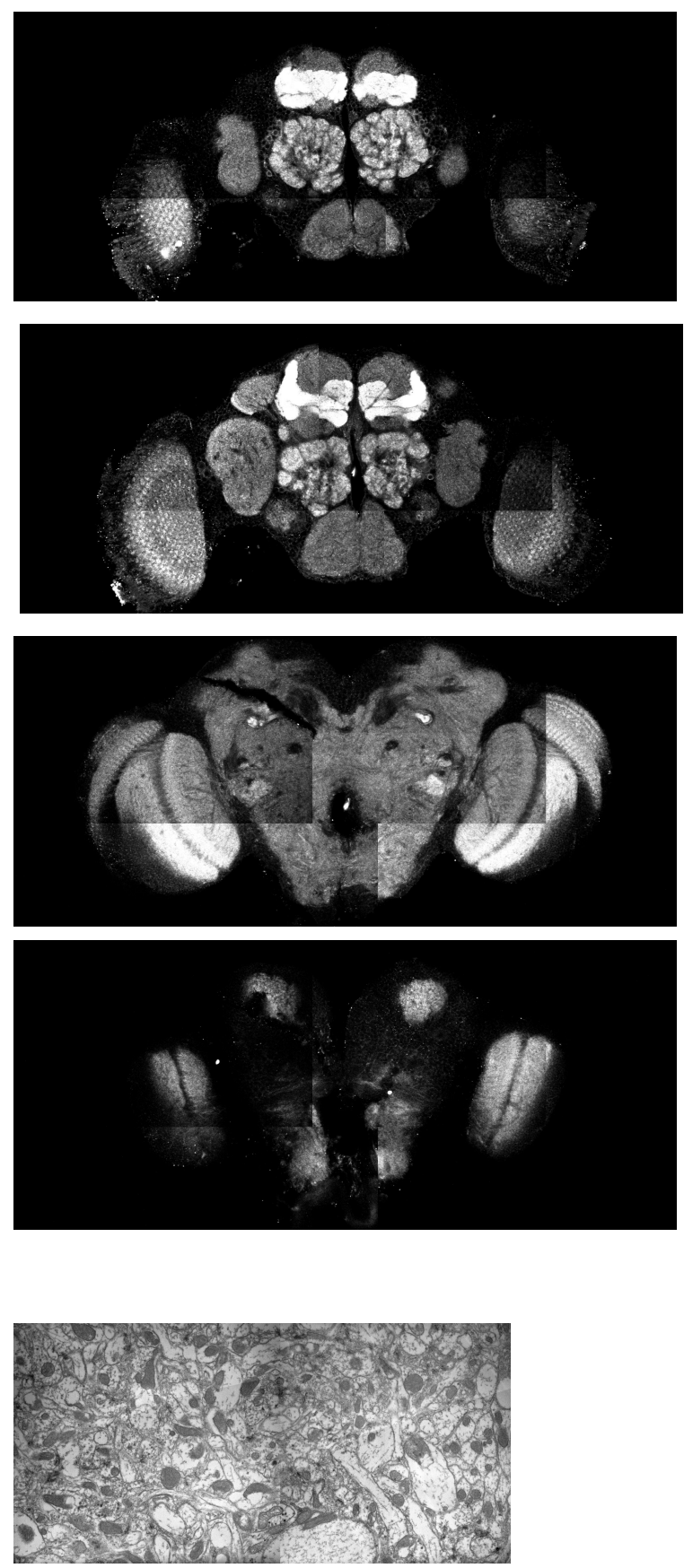

Figure 8: Left column: the mosaic results derived by our method on images at different depths. Right column: the direct stitching results. From top to bottom: the $24^{\text {th }}$ slice of the whole image stack, the $32^{\text {nd }}, 94^{\text {th }}, 112^{\text {th }}$ slices and an EM image of Drosophila's neuron fibers. The dimensions of the mosaiced confocal microscope images are $2274 \times 992$, and the dimensions of the EM image are $3520 \times 1696$. The properties at different depths of the images are distinct. Briefly, in the $24^{\text {th }}$ and $32^{\text {nd }}$ slices, the contours of different tissues are clear; and in the $94^{\text {th }}$ slice, the intensity of most areas is homogeneous, except the regions of the two oval-shaped optical lobes, which are much brighter. Finally, the brightness and contrast in the $112^{\text {th }}$ slice are quite low. 

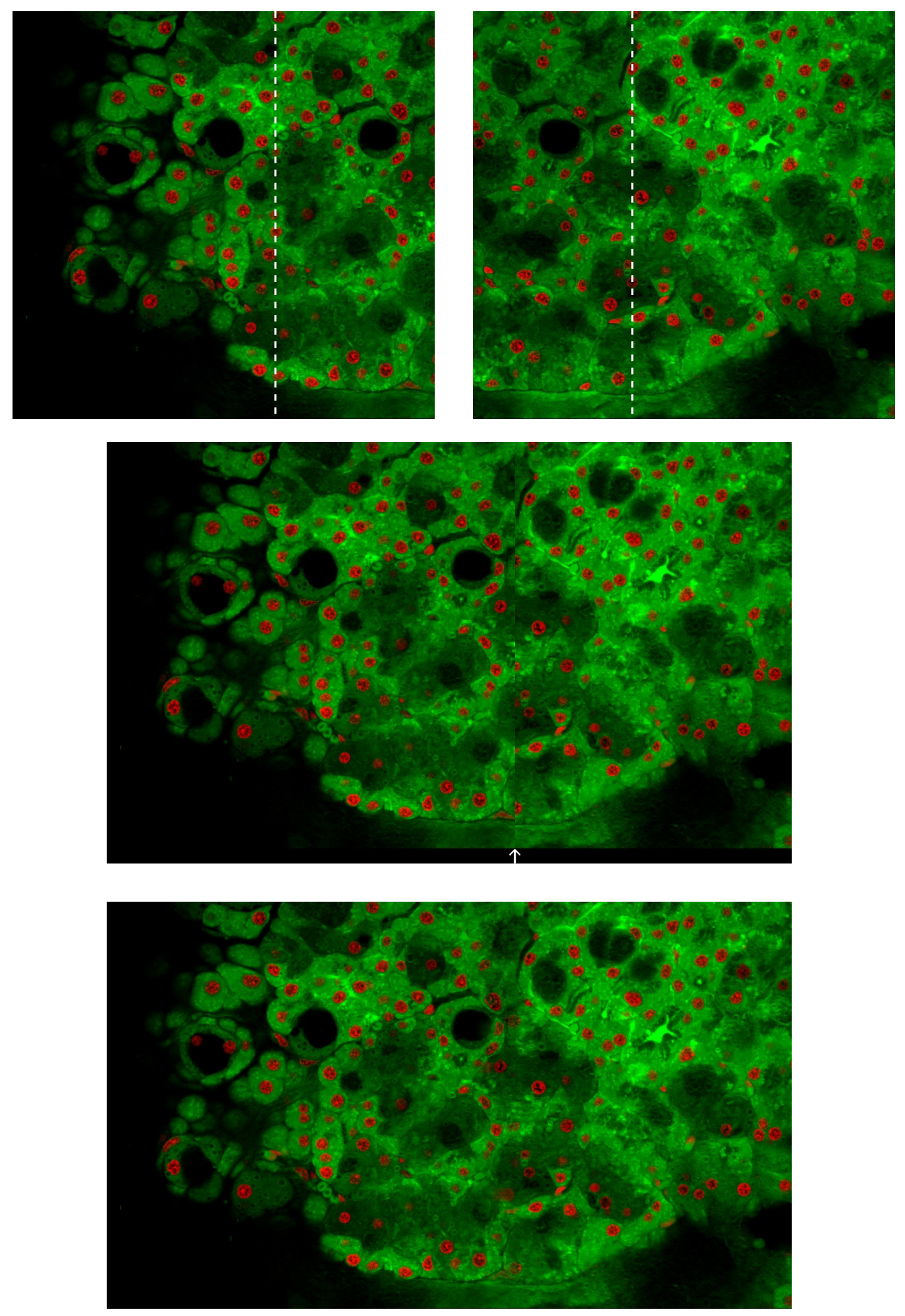

Figure 9: Combining microscope images of mouse pancreases . Top: two images to be combined. The dashed-lines denote the boundaries of the overlapping regions. Middle: the image combined directly without blending. The arrow indicates the selected blending position. Bottom: the mosaic image obtained by the proposed method. 


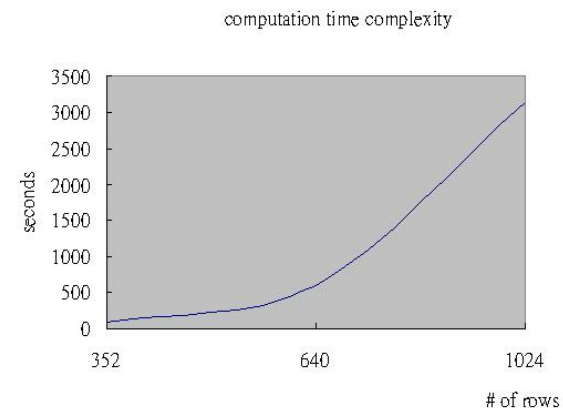

Figure 10: The average computation time of our method versus the number of rows of to-be-combined images. When the number of rows is less than 640 , the time complexity is approximately constant; however, the average time complexity increases when the number of rows in the images is higher. Methods designed to reduce the computational complexity when the number of rows is large are discussed in the text. 